\title{
Improvement of Accuracy of Human Blood Groups Determination using Image processing Techniques
}

\author{
Mehedi HasanTalukder', Md. Mahfuz Reza ${ }^{2}$, Mahbuba Begum ${ }^{3}$, Md. Rabiul Islam ${ }^{4}$, Md. Mahmudul Hasan
}

Dept of Computer Science and Engineering, MawlanaBhashani Science and Technology University (MBSTU) Santosh,

Tangail, Bangladesh ${ }^{1,2,3,4,5}$

Abstract: It is very crucial to determine human blood groups in an emergency situation. But according to current system, the detection procedure is very slow. At present, human blood groups are determined manually through plate test procedure. It consists of blood collection and mixing with specific reagents in order to determine the blood agglutination. The results are checked microscopically. In this paper, the main objective is to present a methodology to determine human blood groups using image processing techniques.

Keywords: Blood types, Image processing, Standard deviation, Plate method, Agglutination, MATLAB, Accuracy, Human Blood, Antigens.

\section{INTRODUCTION}

In Bangladesh, according to [1], more than 4000 people are died due to the frequent road accidents and work accidents every year. In these accidents it is often necessary to perform urgent blood transfusion where it is essential to determine blood group of the victim rapidly. In real time, the health technicians, in these situations, must decide quickly what procedures they must apply, in order to guarantee the best treatment for the patient. In the mentioned emergency situations, where there is no time for human blood typing, the universal donor blood is administrated. As a result, some reactions may occur, risking the patient's life $[2,3]$ and stock levels of blood from universal donor blood type decreases. Now-a-days, human blood groups are determined using two methods in our medical system, named plate method and card method. In plate method, three drops of human blood samples are taken in three plates and anti-A, anti-B anti-D reagents are mixed with them respectively. Then observing whether agglutination occurs or not, the human blood groups are determined according to the following:

\begin{tabular}{|c|c|c|c|c|}
\hline Reagent & $\begin{array}{c}\text { Anti- } \\
\mathrm{A}\end{array}$ & Anti-B & $\begin{array}{c}\text { Anti- } \\
\mathrm{D}\end{array}$ & $\begin{array}{c}\text { Blood } \\
\text { groups }\end{array}$ \\
\hline \multirow{5}{*}{ Agglutination } & $\sqrt{ }$ & $\times$ & $\sqrt{ }$ & $\mathrm{A}^{+}$ \\
\cline { 2 - 5 } & $\sqrt{ }$ & $\times$ & $\times$ & $\mathrm{A}^{-}$ \\
\cline { 2 - 5 } & $\times$ & $\sqrt{ }$ & $\sqrt{ }$ & $\mathrm{B}^{+}$ \\
\cline { 2 - 5 } & $\times$ & $\sqrt{ }$ & $\times$ & $\mathrm{B}^{-}$ \\
\cline { 2 - 5 } & $\sqrt{ }$ & $\sqrt{ }$ & $\sqrt{ }$ & $\mathrm{AB}^{+}$ \\
\cline { 2 - 5 } & $\times$ & $\sqrt{ }$ & $\times$ & $\mathrm{AB}^{-}$ \\
\cline { 2 - 5 } & $\times$ & $\times$ & $\times$ & $\mathrm{O}^{+}$ \\
\hline
\end{tabular}

Table 1: Possible types of agglutinations [4]

The card method [5], requires mixing the sample of blood with the context of the micro tubes available in the cardID, followed by a centrifugation and the results interpretation. Though, it is accurate approach, it has the disadvantages of requiring 30 minutes, which is excessive especially in emergency situations.
Considering the disadvantages of the card method, we will work with plate method in this paper.

\section{ALGORITHM FOR DETERMINATION OF HUMAN BLOOD GROUPS}

To determine the blood groups using image processing techniques we have to perform the following steps:

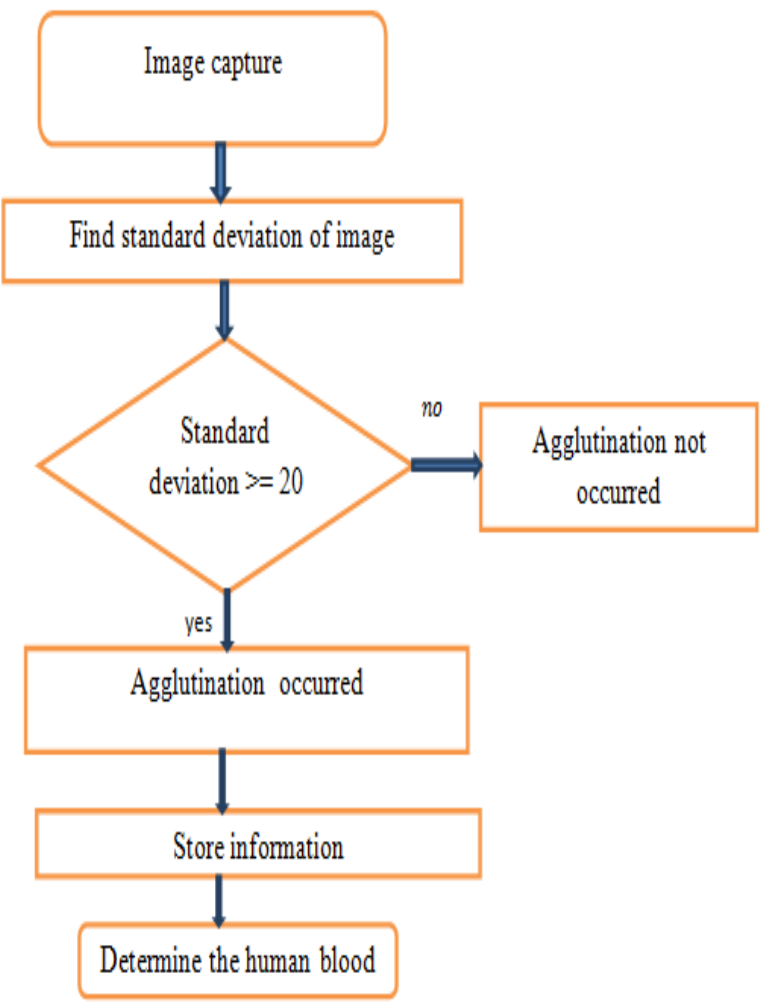

Fig 1: Flowchart of human blood groups determination

Considering several tests performed, it was verified that when agglutination occurs in the blood-antigen mixtures, the standard deviation values of the analysed image are greater than 20 [6]. 


\section{International Journal of Advanced Research in Computer and Communication Engineering}

\section{Vol. 4, Issue 10, October 2015}

With this data it is possible to correctly classify the blood groups.

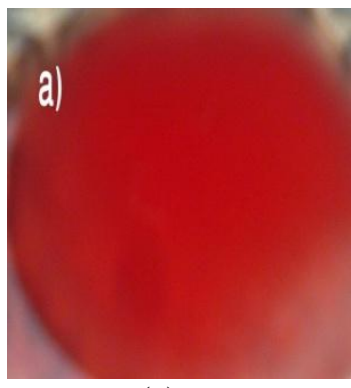

(a)

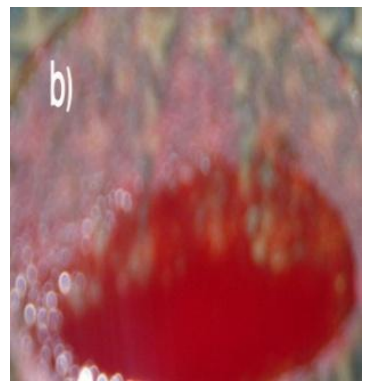

(b)
Figure 2: a) Presence of agglutination b) Absence of agglutination.

The standard deviation [8,9], is the parameter that allows distinguishing with superior exactness and effectiveness the occurrence or not of agglutination.

We calculate the standard deviation (SD) using MATLAB according to the following equation:

$$
\mathrm{SD}=\sqrt{\frac{1}{N} \sum_{i=1}^{N}\left(x_{i}-\mu\right)^{2}}
$$

Where,

$\mathrm{N}=$ Total number of values

$x_{i}=\mathrm{i}_{\mathrm{th}}$ Position value

$\mu=$ Mean value

\section{III.EXPERIMENTAL RESULT}

The methodology described in the above was applied to a set of images with the same blood type, using the three different antigens of test.

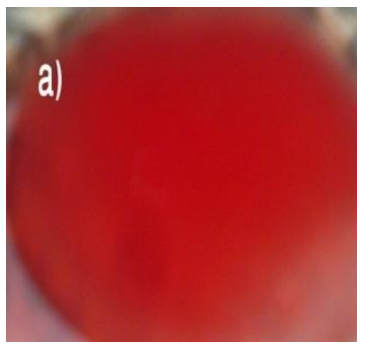

(a)

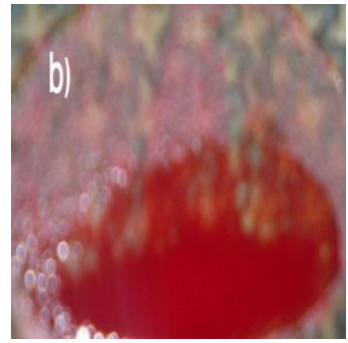

(b)

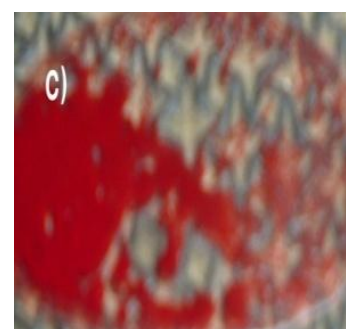

(c)

Figure 2: a) Blood image with anti-A,b) Blood image with anti-B c) Blood image with anti-D

Analyzing the above table, it is verified that agglutination does not occur in Figure 2a) because the standard deviation is less than 20. But occurs in figure $2 b$ ) and figure 2c) as standard deviation is greater than 20. Here Anti-A and Anti-B determine the human blood type $(\mathrm{A}, \mathrm{B}, \mathrm{AB}, \mathrm{O})$ and Anti-D determines whether the blood group is positive or negative. In the above figure $2 \mathrm{a}$ ), antiA does not react with blood. In figure $2 b$ ) anti-B reacts with blood. That is the blood type is $\mathrm{B}$. In figure $2 \mathrm{c}$ ) the anti-D reacts with blood, so it is positive type blood. Thus considering the results obtained, it is concluded that the blood type of the analyzed blood is $\mathrm{B}^{+}$.Thus, applying this method, we can determine other blood groups.

The standard deviation of the above images is as follows:

Table 2: Standard Deviation of the above Images

\begin{tabular}{|c|c|c|}
\hline Figure & Antigens & $\begin{array}{c}\text { Standard } \\
\text { deviation }\end{array}$ \\
\hline 2a) & Anti-A & 16.80183 \\
\hline 2b) & Anti-B & 31.26805 \\
\hline 2c) & Anti-D & 51.17779 \\
\hline
\end{tabular}

\section{IV.CONCLUSIONS AND FUTURE WORK}

Considering the studies presented in this work, it can be concluded that the applied image processing technique enables determining fast accurately the blood type of the analyzed samples of blood. Clearing distinct zones in the pixels intensity of the images are identified, allowing classifying with mathematical basis quantification the agglutination occurrence. As a reference, it was the plate method, adjusted conveniently to the methodology of detection of blood type using image processing, presenting safe results in a time inferior to 2 minutes, thus, the use of the approach described in this work allows eliminating the errors committed by the technicians in the blood type classification. This will contribute to undertake safe blood transfusions and to reduce the loss of human lives.

\section{REFERENCES}

[1] http://www.guardian.co.uk/global-development/2012/aug/22 /bangladesh-road-accidents-poor-economy.

[2] Wong, B.L.W., Blandford, A., "Naturalistic Decision Making in Emergency Ambulance Command and Control" (Discussion Paper Series No. 2001/11). Department of Information Science, University of Otago, Dunedin. 2001.

[3] National Institute of Medical Emergency. INEM, Available at < http://www.inem.pt > Accessed on:16-04-2012.

[4] Method of human blood types determination: http://www.prof2000.pt/users/csilvana/Metod.html. Accessed in June 2012

[5] Diagnostic and Medical Products[Internet homepage].[accessin 2009 April] Availablein:www.diamed.ch/product_detail. aspx? id=92\&navvis=Datasheet of Diamed-ID, 2008.Card-ID.Diaclon $\mathrm{ABO} \backslash \mathrm{Rh}$ for patients.Cressier

[6] Ana Ferraz, VítorCarvalho and PatríciaBrandao "Automatic Determination of Human Blood Types Using Image Processing Techniques", 3 4750-117 Arcozelo, Barcelos, Portugal.

[7] A. Ferraz, V. Moreira, D. Silva, V. Carvalho and F. Soares, "Automatic system for blood type classification using image processing techniques", Biodevices 2011, Rome, Italy, 26-29 January 2011.

[8] http://www.mathsisfun.com/data/standard-deviation.html

[9] Gonzalez R.C., Woods R.E., Digital Image Processing, Upper Saddle River, NJ Prentice Hall,2008

[10] Wong, B.L.W., Blandford, A., "Naturalistic Decision Making in Emergency Ambulance Command and Control" (Discussion Paper Series No. 2001/11). Department of Information Science University of Otago, Dunedin. 2001 